\title{
Movimientos de la corteza y gravedad
}

\author{
E. RODRÍGUEZ PUJOL \\ Instituto Geográfico Nacional. Universidad de Alcalá de Henares \\ erpujol@yahoo.es
}

Recibido: $12 / 06 / 2011$

Aceptado: 25/05/2011

\section{Resumen}

Las tasas intersísmicas de variación temporal de la gravedad en los ámbitos global, regional y local difieren si se miden en zonas sismotectónicamente activas y en zonas sismotectónicamente estables. La gravedad en la superficie terrestre varía de forma temporal secularmente debido principalmente a los movimientos verticales lentos de la corteza terrestre, en fallas tectónicas y especialmente, en las regiones cercanas a los bordes de las placas tectónicas. En zonas estables las variaciones son del orden de 0-0,4 microGal/año $(0-1,2 \mathrm{~mm} / \mathrm{año})$ y en zonas activas se ha cuantificado en tasas de 0,6-4 microGal/año (1-12 mm/año). Las medidas gravimétricas absolutas pueden proporcionar información valiosa sobre los movimientos verticales intersísmicos de la corteza terrestre.

Palabras clave: gravedad, corteza, geodinámica, series temporales, sismotectónica, GPS, Geodesia.

\section{Crustal movements and gravity}

\begin{abstract}
Gravity time variation interseismic rates differ globally, regionally and locally if they are measured in active regions or in stable regions. In the long run, gravity in the surface of the earth changes with time mainly due to the slow vertical movements of the earth crust, tectonic faults and especially, in regions close to the plate boundaries. In non-active regions gravity change rates are about $0-0.4 \mathrm{microGal} / \mathrm{year}$ $(0-1.2 \mathrm{~mm} /$ year) while unveiling in active regions rates about $1-4 \mathrm{microGal} /$ year $(2-12 \mathrm{~mm} /$ year$)$. Absolute gravity measurements can give us valuable information about interseismic vertical displacements of the earth crust.
\end{abstract}

Keywords: gravity, crust, geodynamics, time series, seismotectonics, GPS, Geodesy.

Sumario: Introducción. 1. Movimientos verticales de la corteza. 2. Cambios de gravedad con origen geodinámico. 3. Cambios globales de gravedad. 4. Cambios regionales de gravedad. 5. Cambios locales de gravedad. 6. Conclusión. Referencias bibliográficas.

\section{Referencia normalizada}

Rodríguez Pujol, E., (2011). Movimientos de la corteza y la gravedad. Física de la Tierra Vol. 23 Núm. 1 (2011): 149-171

\section{Introducción}

Los movimientos de la corteza terrestre se pueden deducir por evidencias geológicas, evidencias geomorfológicas y por métodos geofísicos y geodésicos (Scheidegger, 1986). Los estudios sismotectónicos intentan integrar los datos que nos proporcionan los terremotos con otra información disponible a partir de la tectónica, geofísica o geología de una región particular (Udías, 1999), para explicar los procesos litosféricos y el comportamiento de los límites de placas y las fallas activas. La determinación de la posición ha experimentado con las modernas 
técnicas por satélite y otras, una precisión desconocida en la Geodesia clásica. Los principios relativos a cada una de estas técnicas están descritos en Torge (2001) y Seeber (1993). Existen diversas técnicas para discernir cuál puede ser el movimiento de las placas tectónicas o de los procesos tectónicos en cada región de la Tierra: la interferometría de muy larga base (VLBI, "Very Long Base Interferometry"), la distanciometría por satélite (SLR, "Satellite Laser Ranging"), y el sistema de posicionamiento global (GPS, "Global Positioning System"), entre otros. Los movimientos tienen distinta amplitud según sean globales, regionales o locales. La corteza tiene desplazamientos horizontales cosísmicos entre 0-60 m, mientras los intersísmicos oscilan entre $0-15 \mathrm{~cm}$ (e.g. unos $85 \mathrm{~mm} /$ año en la placa Pacífica al este de Honsu, 12-15 cm/año en la placa de Nazca). Los verticales suelen tener un orden de magnitud menor. Desde el siglo XIX, la medida de la gravedad ha evolucionado para producir instrumentos más exactos. La medida de la gravedad en forma absoluta es una de las técnicas más precisas, adecuada para la detección vertical de desplazamientos inter-, pre- y post-sísmicos en la corteza terrestre.

\section{Movimientos verticales de la corteza}

Los movimientos verticales en la corteza se pueden clasificar en lineales o acelerados, o bien continuos o discontinuos. Los lineales corresponden a un movimiento de largo período en la corteza debido a procesos tectónicos, mientras que los acelerados corresponderían a situaciones pre- o post-sísmicas. Se asume a priori que los cambios temporales de la gravedad inducidos por la deformación cortical son graduales, son señales de largo período y con amplitudes pequeñas (Vańiček y Krakiwsky, 1986).

Las tasas verticales de variación de la gravedad y de variación en la altitud se pueden asimilar a las variaciones detectadas mediante observaciones repetidas en el tiempo o series temporales:

$$
\dot{g} / \dot{H} \approx \partial g / \partial H
$$

Según Scholz (1972), los movimientos corticales teniendo en cuenta un ciclo completo de deformación se clasifican en cuatro fases o tipos:

1. Intersísmicos o seculares (asísmicos): casi continuos, tasa constante. Conectados o relacionados con el movimiento de las placas tectónicas.

2. Pre-sísmicos: más rápidos, anteriores a terremotos. Son poco comunes.

3. Co-sísmicos: muy rápidos, salto de falla sísmico.

4. Post-sísmicos: bastante rápidos, después de los terremotos.

Para el caso de grandes variaciones de la gravedad, las cuales se producen eventualmente en zonas volcánicas o cercanas a deformaciones previas o posteriores a terremotos, las señales son mucho más grandes que las incertidumbres instrumentales, aunque no siempre ha sido así. En el caso en el que la señal esperada junto a otras correcciones del entorno sea del mismo orden que las incertidumbres instru- 
mentales, y el número de muestras sea pequeño, estas condiciones hacen que la interpretación de la serie temporal sea difícil (Sasagawa y Zumberge, 1991). Cuando la señal es lo suficientemente dilatada en el tiempo, los efectos estacionales del entorno de la estación son asumibles como ruido si la señal es lo suficientemente amplia, incluso si no hay un considerable número de muestras. Los gravímetros absolutos son especialmente adecuados para este tipo de señal de variación de la gravedad debida a procesos tectónicos de largo período y la pequeña incertidumbre instrumental que se puede conseguir en las medidas (Van Camp et al., 2005), una vez se han tenido en cuenta las correciones de mareas terrestres, movimiento del polo, carga oceánica, carga atmosférica y presión barométrica local (Rodríguez Pujol, E. 2005), fenómenos ajenos al desplazamiento vertical secular tectónico de la corteza (tabla 1).

La técnica GPS es tres veces menos precisa en la coordenada vertical que en las horizontales (Nikolaidis, 2002), esencialmente por razones geométricas, ya que las órbitas de los satélites están lejanas de la superficie de la Tierra. La nivelación geométrica es relativa respecto al origen de altitudes y no es instantánea. Si el origen de altitudes es distinto en cada país, o bien este origen tiene variaciones seculares, no podremos averiguar más que variaciones relativas respecto de ese origen. No está afectada de las variaciones del nivel freático (tabla 1), al igual que el GPS, aunque efectos de carga sí son medibles. La gravimetría es precisa e instantánea y está afectada de los cambios en el nivel freático y su carga.

La nivelación geométrica proporciona altitudes (procedentes de las sucesivas redes de nivelación de alta precisión en España desde 1872), y no puede dar respuesta a señales de ciclos menores que el diurno. Puede ser válida para el largo período si se logra mantener la señalización en el tiempo, las medidas y cálculos son homogéneos, y las precisiones son comparables en las campañas de épocas distintas (Giménez, Suriñach, Goula y Caturla, 1993). Pero siempre hay que recordar que es una medida relativa respecto de un origen elegido mediante convención. Y ese origen también está sujeto a movimiento. No obstante ha sido una primera medida indicativa de los movimientos verticales de la corteza. Así lo demuestran los estudios en el noreste de la Península Ibérica (Giménez, Suriñach, Fleta y Goula, 1996); y en el sur de la Península Ibérica de Giménez, Suriñach y Goula (2000) donde se muestran variaciones verticales de entre 0,9 mm/año y 2 mm/año.

Tabla 1. Correcciones de las distintas técnicas previas a la detección de movimientos corticales.

\begin{tabular}{lccccc}
\hline \multicolumn{1}{c}{ Corrección } & Nivelación & GPS & Gravedad (absoluta) & VLBI & SLR \\
\hline Mareas terrestres & NO & SI & SI & SI & SI \\
Movimiento Polo & NO & SI & SI & SI & SI \\
Carga oceánica & NO & SI & SI & SI & SI \\
Carga Atmosférica & NO & SI & SI & SI & SI \\
Nivel freático & NO & NO & NO & NO & NO \\
\hline
\end{tabular}


A partir de anomalías de la gravedad para la obtención del geoide gravimétrico, las depresiones del geoide pueden indicar levantamientos de la corteza, como ocurre en el rebote post-glacial Báltico de $9 \mathrm{~mm}$ /año (Bjerhamar, 1981). El geoide puede estar relacionado con la cinemática de las placas tectónicas. Existe una tendencia a disminuir suavemente la ondulación del geoide a medida que nos alejamos de zonas de creación de corteza en las dorsales oceánicas. En zonas de subducción y colisión entre placas se produce un aumento más pronunciado de la ondulación del geoide (Jacoby y Seidler, 1981).

El estudio de las anomalías de la gravedad puede proporcionar importante información sobre la composición y grosor de la corteza. También algunos autores han deducido de ellas información sobre movimientos que se producen en la misma. Los grandes gradientes de gravedad pueden indicar transiciones laterales de rocas de alta densidad a rocas de baja densidad o viceversa. Las dos zonas de anomalías positivas de máximos $110 \mathrm{mGal}$ y $80 \mathrm{mGal}$ al Suroeste y al este respectivamente de Málaga, y que también se producen en la costa de Beni Bouchera de Marruecos (80 $\mathrm{mGal}$ ), son asignadas a rocas ultrabásicas y metamórficas (peridotitas), puesto que coinciden parcialmente con afloramientos. Dichas anomalías junto con los acusados gradientes de gravedad indicarían discontinuidades de densidad de gran inclinación (Bonini et al., 1973). Anomalías de aire libre positivas indicarían excesos de masa o tendencia a la subsidencia (Morelli et al., 1975). Las mismas anomalías se encuentran en los mapas más recientes pero en sistema de IGSN71/GRS67 (Liu, 1993; Torné, 2000). En el centro del mar de Alborán existe un máximo $90 \mathrm{~km}$ al este del Estrecho de Gibraltar que se extiende hacia el este hasta el eje de anomalías positivas máximas del Mar Mediterráneo (Morelli, 1980), y que coincide con el aumento de gradiente geotérmico (Torné et al., 2000).

\section{Cambios de gravedad con origen geodinámico}

Las variaciones de gravedad detectadas mediante observaciones repetidas en el tiempo representan información importante de los desplazamientos terrestres de masas, una vez se han eliminado las variaciones temporales provenientes de cambios de masas extraterrestres (movimiento Lunar, Solar y de otros planetas del sistema solar como Júpiter) que se conocen como mareas terrestres. La aceleración de la gravedad es función de la constante de gravitación universal $\mathrm{G}$, de la rotación de la Tierra $\omega$, de la posición donde se realiza la medida $\mathbf{r}$ y de la densidad de las fuentes generadoras del campo gravitatorio $\rho\left(\mathbf{r}^{\prime}\right): g=g\left(G, \omega ; r, \rho\left(r^{\prime}\right)\right)$. Si G y $\omega$ se consideran constantes, entonces conociendo el gradiente $\delta g / \delta$, la expresión siguiente muestra que los cambios temporales de gravedad contienen el efecto combinado de variaciones en la altitud y variaciones en la densidad:

$$
\dot{g}=(\partial g / \partial t)=(\partial g / \partial r) \cdot(\partial r / \partial t)+G \iiint\left(\rho\left(r^{\prime}\right) /\left(r^{\prime}-r\right)^{2}\right) d v
$$




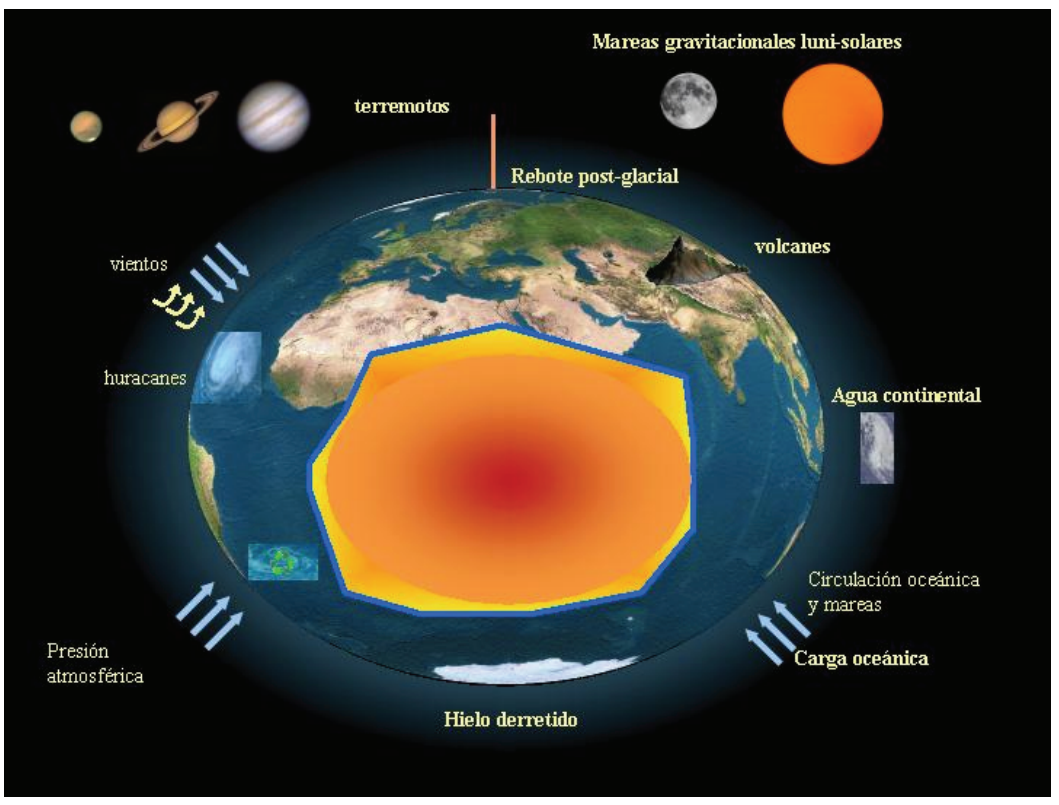

Figura 1. Fenómenos de cambios de masa que afectan a la Tierra (adaptado de Lambert y Chao).

La gravedad se puede considerar como una variable ambivalente: es un detector tanto de movimientos verticales como de cambios de masa (figura 1). Los cambios en el módulo de la aceleración vertical de la gravedad en la superficie de la corteza de la Tierra pueden ser debidos no solamente a la variación de la distancia entre el centro de masas de nuestro planeta y el punto en el que se realiza la medición, sino también debidos al movimiento relativo de masas dentro de la Tierra. Con el objetivo de obtener estos tipos de cambios de la gravedad se pueden utilizar varios observables: el potencial gravitatorio y su primera, segunda y tercera derivadas; las mareas terrestres y sus constituyentes, el propio valor absoluto de la gravedad, gradientes verticales y horizontales, anomalías y perturbaciones, por medio de perfiles gravimétricos, redes y gravedad absoluta. En general, las variaciones temporales de la gravedad tienen una amplitud y frecuencia que depende del fenómeno considerado. Dependiendo del área de conocimiento objeto de estudio, unos fenómenos serán considerados como ruido, o bien como señal. En el caso de ruido deberán ser corregidos, acotados o despreciados según afecten o no a la señal objeto de estudio. Algunos de ellos se superponen en periodo, por lo que no es sencillo el poder diferenciarlos (i.e. carga oceánica y cambios del nivel freático). En la figura 2 se muestran las variaciones de gravedad con el tiempo en el eje de ordenadas, mientras que en el eje de abscisas se muestran los períodos. 


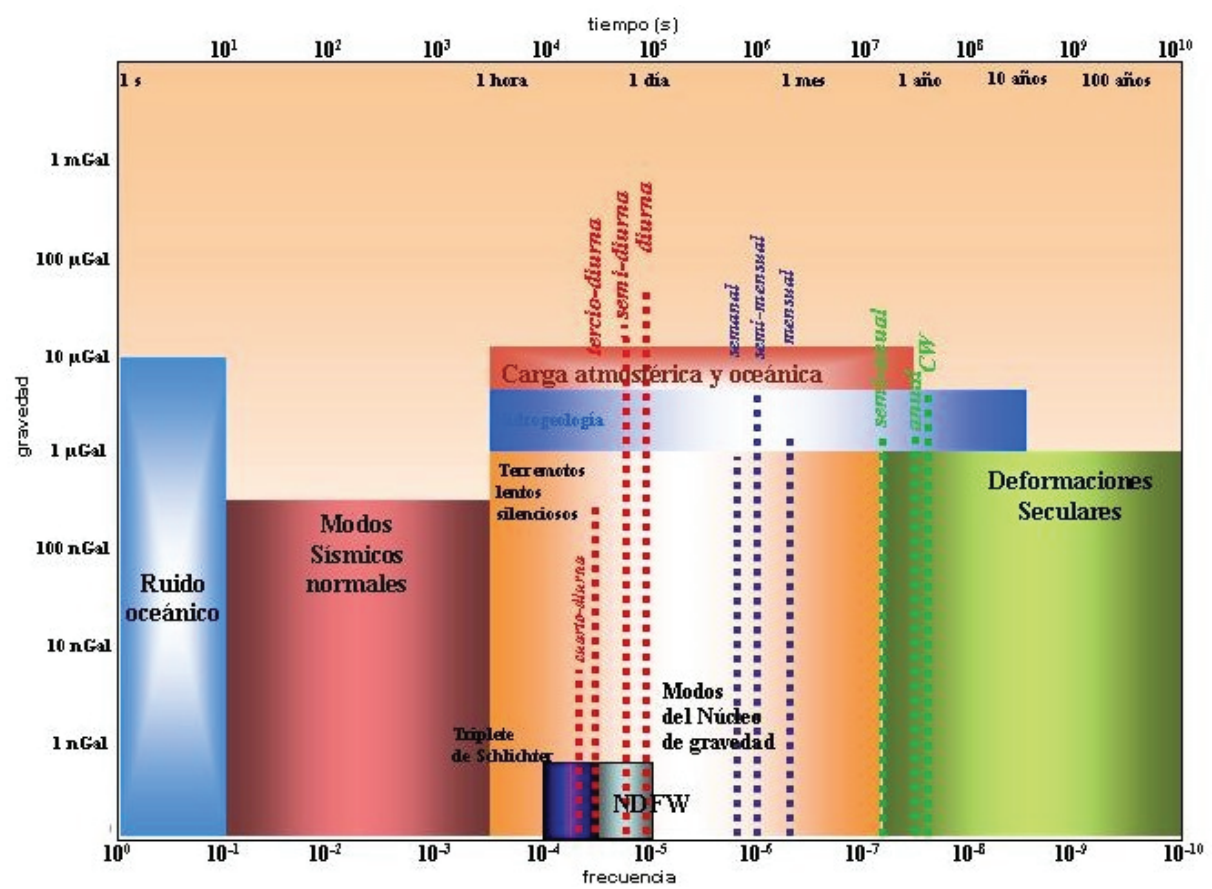

Figura 2. Frecuencias, períodos y amplitudes en las variaciones de gravedad con el tiempo (adaptado de Hinderer y Dittfeld).

\section{Cambios globales de gravedad}

Podemos citar como cambios globales en el valor de la gravedad en todas las partes del globo aquellos que tienen su origen en circunstancias de una extensión suficientemente grande como para cambiar el campo gravitatorio total o crear algún tipo de variación global. Afectan por tanto a los coeficientes armónicos del desarrollo en serie del campo gravitatorio.

Si la masa de la Tierra es M, G la constante de gravitación universal, a el radio ecuatorial de la Tierra, $\vartheta$ la colatitud y $\lambda$ la longitud, el potencial gravitatorio terrestre $\mathrm{U}$ en coordenadas esféricas $(r, \vartheta, \lambda)$ con origen en el centro de masas de la Tierra se puede expresar como:

$U=\frac{G M}{r}\left\{1-\sum_{n=2}^{\infty}\left(\frac{a}{r}\right)^{n} J_{n} P_{n}(\cos \theta)+\sum_{n=2}^{\infty} \sum_{m=1}^{n}\left(\frac{a}{r}\right)^{n} P_{n}^{m}(\cos \theta)\left(A_{n m}(\cos m \lambda)+B_{n m}(\operatorname{sen} m \lambda)\right)\right\}$

siendo las funciones $\mathrm{P}_{\mathrm{n}}(\cos \vartheta)$ los polinomios de Legendre de orden $\mathrm{n}$ y $\mathrm{P}_{\mathrm{n}}{ }^{\mathrm{m}}(\cos \vartheta)$ los polinomios asociados de Legendre (soluciones particulares de la ecuación de Laplace), respectivamente. Los momentos de gravitación, números adimensionales

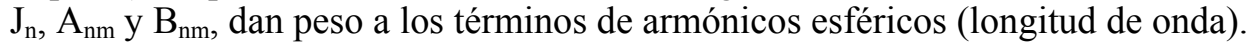


La distinta orientación del eje de rotación del manto con respecto al núcleo terrestre el NDFW ("Nearly Diurnal Free Wobble"), así como del núcleo externo respecto del núcleo interno (FCN, "Free Core Nutation"), pueden dar lugar a variaciones globales que se encuentran por debajo del $\mu \mathrm{Gal}$, detectables con los gravímetros superconductores. Estos son los modos normales del núcleo, cuyo primer modo se denomina triplete de Schlichter. Por ejemplo, los desplazamientos de masas convectivas (agua, atmósfera, manto) de muy largo período en el interior de la Tierra (figuras 1 y 2) pueden afectar a los primeros armónicos del potencial gravitatorio (tablas 2 y 3 ), especialmente a $J_{2}$, o producir variaciones del vector de rotación de la Tierra. Las anomalías de la gravedad de muy larga longitud de onda pueden ser causadas por las plumas ascendentes o descendentes de material del manto convectivo.

Otro ejemplo interesante lo encontramos en las variaciones inducidas por terremotos muy grandes en la variación del coeficiente $J_{2}=-2 \cdot 10^{-12}$ entre los años 1977-1985 (Chao y Gross, 1987). Las variaciones detectadas mediante gravímetros criogénicos han permitido evaluar de nuevo los modos normales de oscilación de la Tierra. Se han realizado cálculos sobre su posible influencia sobre el eje instantáneo de rotación de la Tierra con motivo de terremotos de magnitud superior a 8 (Chile 1960, Sumatra 2004, Chile 2010, Hosuko-Oki 2011). Las observaciones absolutas y relativas de la gravedad también detectan estas perturbaciones mediante los gravímetros absolutos y relativos.

Tablas 2 y 3 . Variaciones seculares $\left(10^{-11} /\right.$ año $)$ en los coeficientes zonales pares e impares bajos del campo gravitatorio terrestre a partir de medidas de SLR.

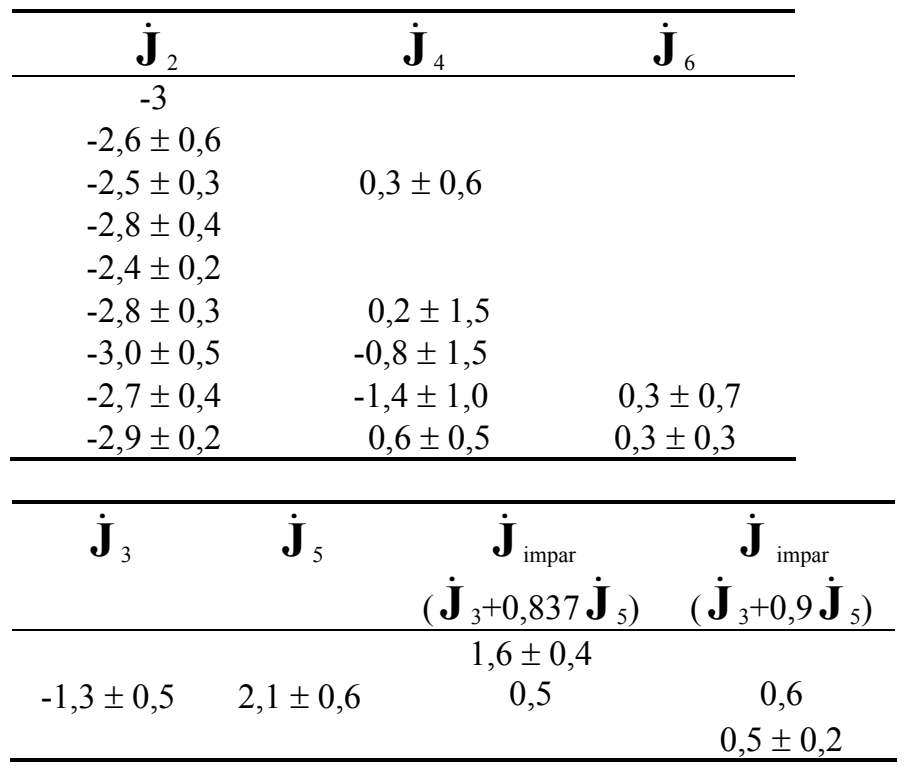


Las últimas observaciones por satélite del campo gravitatorio terrestre han sido posibles gracias a los proyectos GRACE ("Gravity Recovery and Climate Experiment"), CHAMP ("Challenging Mini-satellite Payload"). Las anomalías de gran longitud de onda y su variación con el tiempo son detectables, aunque con menor exactitud que las mediciones realizadas sobre la superficie con los gravímetros absolutos y superconductores. A partir de 14 meses de observaciones de satélite, la Universidad de Texas ha realizado un modelo de campo gravitatorio global con 200 términos denominado "GRACE Gravity Model 02" (GGM02C), combinando datos de satélites gemelos (GRACE) y terrestres. En Europa, el GFZ ("GeoForschungZentrum") de Potsdam ha desarrollado modelos de campos gravitatorios desde el EIGEN-GL01C hasta el nuevo modelo de campo gravitatorio terrestre EIGENGL04C (2006), que resuelve anomalías en el geoide y gravedad de hasta $110 \mathrm{~km}$ de longitud de onda y que contiene términos de desarrollo del potencial hasta el orden y grado 360, uniendo datos del satélite GRACE, Lageos y datos de medida de gravedad en superficie. El modelo previo, EIGEN-GL03C, que unía datos de GRACE y CHAMP, tiene exactitudes de $30 \mathrm{~cm}$ y $8 \mathrm{mGal}$ en resolución de longitudes de onda de $100 \mathrm{~km}$. El proyecto GOCE ("Gravity Field and steady-state Ocean Circulation Explorer"), que comenzó en 2007, obtuvo anomalías de la gravedad con exactitudes de $1 \mathrm{mGal}\left(10^{-5} \mathrm{~ms}^{-2}\right)$ y un geoide con exactitud de 1-2 $\mathrm{cm}$, ambos en resoluciones espaciales mejores de $100 \mathrm{~km}$. Anmalías de gravedad cosísmicas de 15 y 5 microGal se detectaron para los terremotos de Sumatra-Andaman y Chile, respectivamente (De Linage et al., 2009; Heki yMatsuo, 2010).

\section{Cambios regionales de gravedad}

Los cambios regionales en el tiempo de la gravedad son los que afectan a regiones entre cien y varios miles de kilómetros. Tienen también un carácter de muy largo período o tendencia clara, aunque en los procesos de las dorsales oceánicas sean más rápidos. Se producen en los límites de placas, con la acumulación de esfuerzos en mucho tiempo y su liberación. También se producen en las zonas intraplaca debidos al rebote post-glacial, compactación sedimentaria y la neotectónica regional. En zonas volcánicas o de terremotos cambios locales pueden superponerse con los cambios regionales.

En lugares tan activos como las dorsales oceánicas es natural observar grandes variaciones de la gravedad en amplitud y en relativamente corto período de tiempo, asociadas a fenómenos de creación de corteza litosférica ("rifting"). Levantamientos regionales y disminución de gravedad en los flancos del rift, subsidencia local e incremento de gravedad en la zona central de la fisura fueron caracterizados para Islandia con medidas entre 1965 y 1980 (Torge y Kanngieser, 1985). Variaciones en esta zona de hasta $80 \mu \mathrm{Gal}$ en la fisura de Krafla a lo largo de $15 \mathrm{~km}$.

En la zona de Beijing-Tianjin (Kuo y Sun, 1993) y relacionadas con terremotos, se han obtenido variaciones de la gravedad en 59 estaciones realizadas con gravímetros relativos Lacoste-Romberg. 


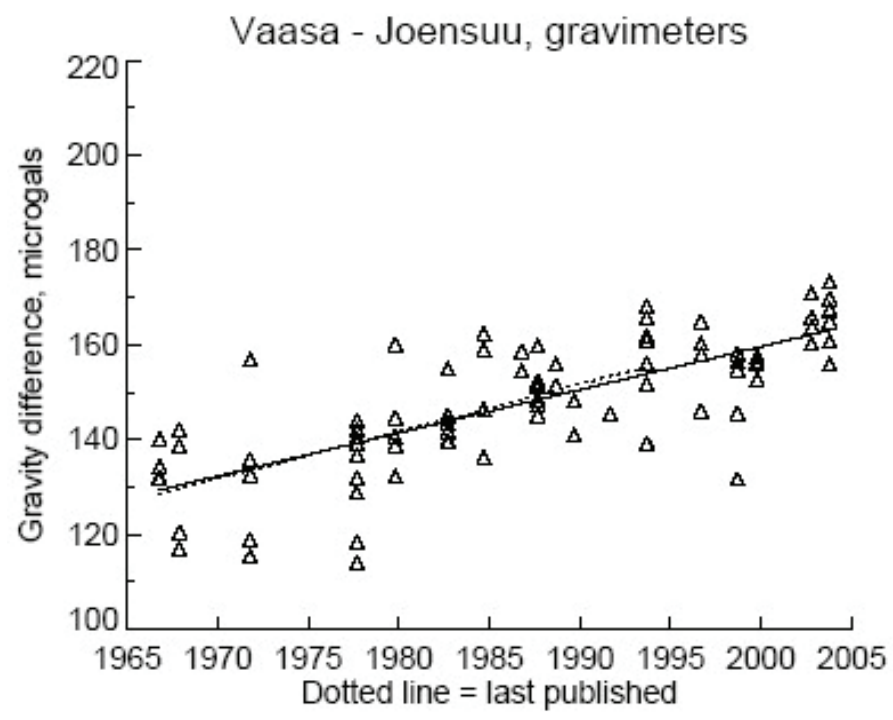

Figura 3. Cambios de gravedad de origen geodinámico en la línea este de Finlandia (tramo Vaasa-Joensuu) obtenidos a partir de medidas relativas de gravedad (Mäkinen et al., 2005).

Jachens y Roberts (1985) observaron una disminución de la gravedad debida a un levantamiento entre 1980 y 1983 en la Caldera de Long Valley después de la serie de terremotos de enero de 1983, y que probablemente fue seguida de adición de masa en profundidad. Los modelos propuestos incluyen inyección de magma a profundidades entre 3 y $5 \mathrm{~km}$, e implican volúmenes inyectados de hasta $0,2 \mathrm{~km}^{3}$ (Rundle y Whitcomb, 1986). La gravedad y sus anomalías pueden cartografiar las distribución de densidad en profundidad e inferir la geología del subsuelo, y los cambios temporales de la gravedad pueden permitir evaluar la tectónica y la actividad volcánica. Se correlacionaron por Jachens y Roberts (1985) máximos de anomalías residuales isostáticas con la región de sismicidad de los enjambres de 1983.

Sin embargo, los dos ejemplos más claros de áreas de levantamiento de la corteza terrestre son la región alrededor del mar Báltico y de la bahía de Hudson. Con datos provenientes de las investigaciones paleoclimatológicas conocemos que estas zonas estuvieron cubiertas de hielo durante la última glaciación. Ahora se elevan progresivamente y este efecto es interpretado como un rebote de la corteza después de que el hielo se ha derretido hace 10.000 años. Estudios desde 1939 por Kukkamaki, Gutenberg, Walcott y Lambert et al. (2001), este último en la figura 4, han confirmado los desplazamientos posteriormente con técnicas actuales en ambos lugares. El rebote post-glacial ha sido estudiado también utilizando datos GPS (Milne et al., 2001), modelos de Tierra viscoelásticos obtenidos por inversión de observaciones del nivel del mar (Lambeck et al., 1990; Han y Wahr, 1995), o por observaciones de gravedad (Ekman y Mäkinen, 1996). Variaciones de dos $\mu \mathrm{Gal}$ 
para levantamientos de $1 \mathrm{~cm}$ al año son los obtenidos en este último estudio utilizando observaciones relativas entre 1966 y 1993 (figura 3). A partir de la variación anual de $1,0 \pm 0,14 \mu \mathrm{Gal} /$ año (junto con medidas de nivelación o GPS) se obtuvo la relación empírica $\dot{\mathrm{g}} / \dot{H}=-2,0 \pm 0,6 \mu \mathrm{Gal} / \mathrm{cm}$, que fue actualizada a $0,91 \pm 0,09$ $\mu \mathrm{Gal} / \mathrm{año} \mathrm{y} \dot{g} / \dot{H}=-1,6 \pm 0,6 \mu \mathrm{Gal} / \mathrm{cm}$ (Mäkinen et al., 2005).

Utilizando diversos datos de gravímetros absolutos, el Instituto Geodésico Finlandés ha obtenido en las estaciones de Vaasa, Sodankylä y Metsähovi, series temporales con tasas de variación de $-2,2,-3,0 \mathrm{y}-1,1 \mu \mathrm{Gal}$ al año, respectivamente (Bilker et al., 2004).

El estudio es comparable por el realizado por Lambert et al. (2001) en Churchill (Canadá) con gravímetros absolutos (figura 4, derecha). Variaciones totales de unos $14 \mathrm{~cm}$ en 12 años, unos $22 \mu \mathrm{Gal}$, que suponen tasa de variación del orden de los 2 $\mu \mathrm{Gal} /$ año. Los gravímetros absolutos son especialmente adecuados para este tipo de estudios de largo período, pues no tienen deriva como el resto de instrumentos utilizados para la medida de la gravedad.

Uno de los estudios acerca del rebote postglacial con datos multidisciplinares es el de Sato et al. (2006). Las tasas de desplazamientos secular obtenidas a partir de VLBI en el marco de referencia del NNR-NUVEL-1A son $0,2 \pm 0,5 \mathrm{~mm} / \mathrm{año}$, $1,7 \pm 0,5 \mathrm{~mm} / \mathrm{año}$, y $4,8 \pm 1,1 \mathrm{~mm} /$ año en la componente norte, este y vertical, respectivamente. Mediante GPS las tasas de desplazamientos obtenidos respecto del mismo sistema de referencia son $0,2 \pm 0,6 \mathrm{~mm} / \mathrm{año},-2,3 \pm 0,6 \mathrm{~mm} / \mathrm{año}$, y $6,4 \pm 1,5$ $\mathrm{mm} / \mathrm{año}$, respectivamente. Las diferencias entre VLBI y GPS son atribuidas a un error en la escala de marco de referencia GPS que corresponde a 1,6 mm/año en las tasas verticales obtenidas por GPS. A partir de datos de mareógrafos los desplazamientos verticales estimados son $5,2 \pm 0,3 \mathrm{~mm} /$ año. En el análisis de datos de gravedad absoluta en un periodo de 1998 a 2002 en Ny-Ålesund con distintos gravímetros FG5 (figura 4, izquierda) se estima una tasa de variación de $-2,5 \pm 0,9$ $\mu \mathrm{Gal} /$ año. Las observaciones de FG5 se ajustan a una recta teniendo en cuenta las incertidumbres instrumentales. Esta tasa de gravedad es aproximadamente el doble que las estimadas por GPS y VLBI para la componente vertical. 

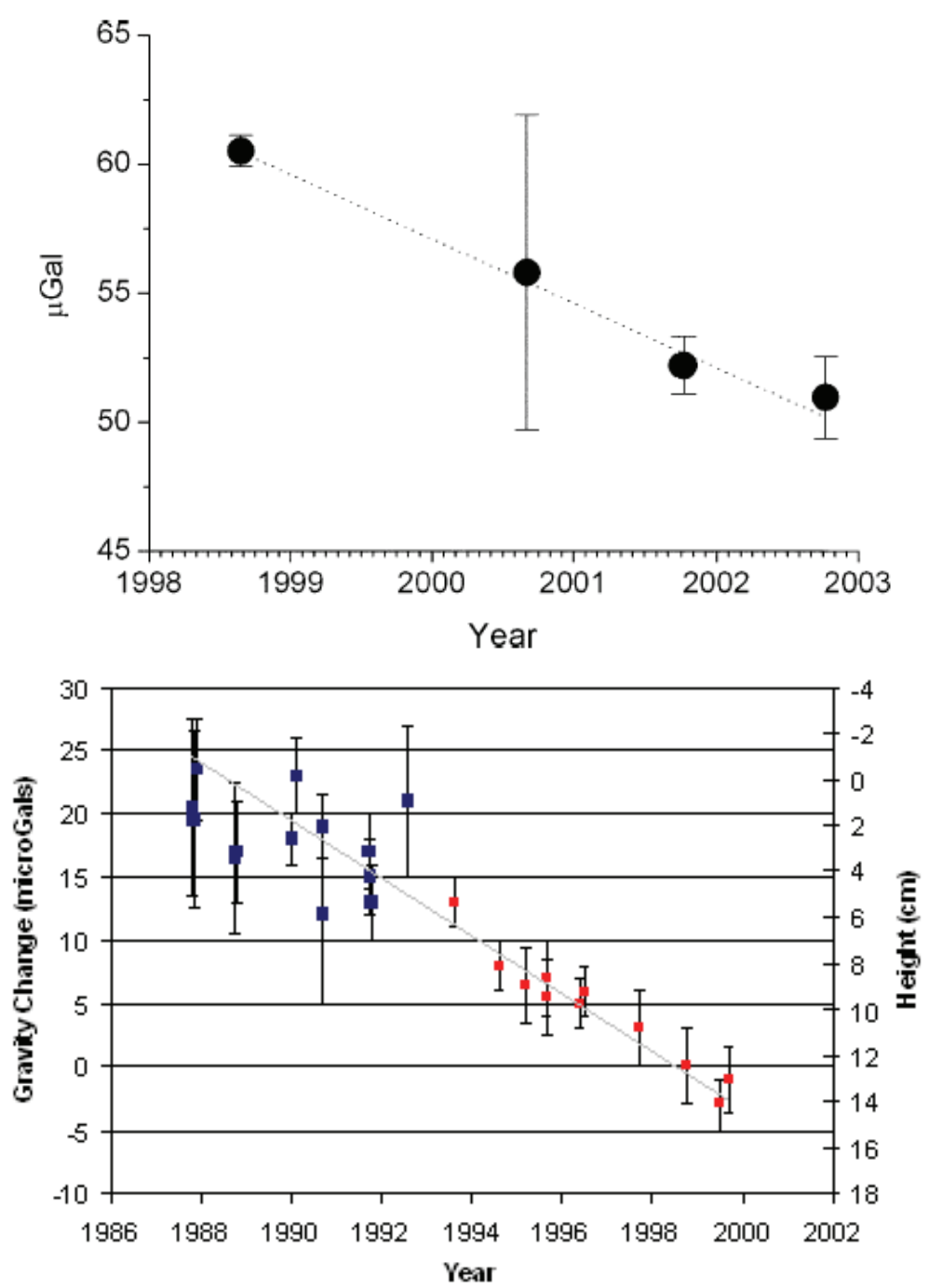

Figura 4. Cambios de gravedad de origen geodinámico en Ny-Ålesund (izquierda, Spitzbergen, Sato et al., 2006) y en Churchill (derecha, Canadá, Lambert et al., 2001), obtenidos mediante medidas absolutas de gravedad.

Las observaciones de gravedad y GPS se pueden comparar con la respuesta elástica existente en la región antártica (Mäkinen et al., 2007). Se contemplan dos modelos que puedan causar movimientos verticales: un fenómeno de rebote elástico post-glacial de mayor largo plazo y otro de rebote elástico producido por el balance más actual de hielo.

Estas observaciones en la Antártida has sido llevadas a cabo por varios autores, entre otros, Cerutti (1992), Hanada (1994), Sasagawa (1993), Mäkinen (1994, 
2004), Almavict (2002), Fukuda (2005), con distintos gravímetros absolutos y están recopiladas en Mäkinen et al. (2007). A partir de estas medidas se han obtenido observaciones repetidas con tasas de variación de gravedad en el continente antártico en estaciones de costa de Aboa, Bahía de Terra Nova, Syowa y McMurdo (figura 5), ya que en el continente las condiciones de observación son difíciles con la instrumentación actual. En el último caso la tasa de variación de 4,2 $\pm 1,4$ $\mu \mathrm{Gal} /$ año se refiere como un poco elevada, mientras que en Terra Nova entre 1995 y 1997 es de $-0,4 \pm 1,5 \mu \mathrm{Gal} / \mathrm{año}$, en Syowa es de $-0,3 \pm 0,4 \mu \mathrm{Gal} / \mathrm{año}$, y en Aboa de $0,5 \pm 0,5 \mu \mathrm{Gal} /$ año.

En general se deduce de las observaciones una estabilidad del continente antártico, aunque se resalta la ausencia de estaciones en el centro del continente. La posible elevación predicha por los modelos de respuesta viscoelástica de la Tierra en esta región a las desglaciaciones pasadas no parece estar refrendada por las observaciones de gravedad y GPS.
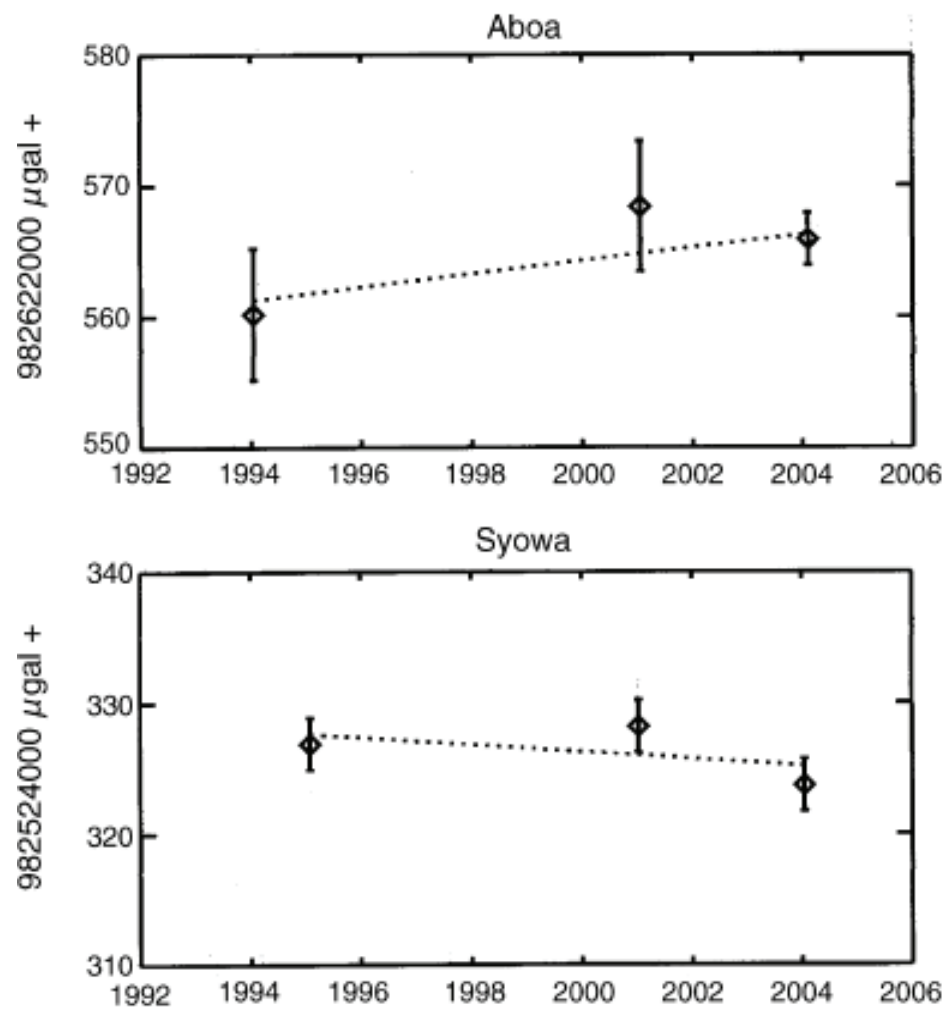

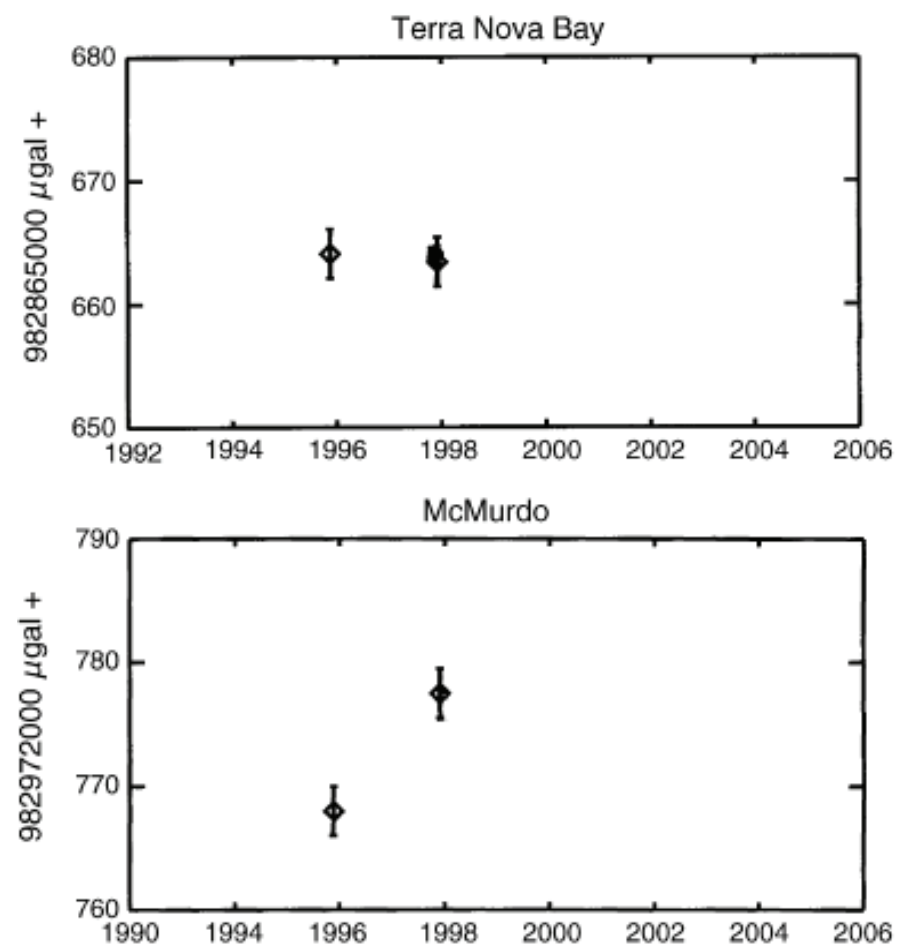

Figura 5. Variaciones temporales de la gravedad expresadas en $\mu \mathrm{Gal}$ en estaciones de la Antártida a partir medidas absolutas de la gravedad en Aboa, Bahía de Terra Nova, Syowa y McMurdo. (Mäkinen et al., 2007).

La tectonofísica de un área activa muestra que cambios de gravedad en el tiempo entre 50 y más de $200 \mu \mathrm{Gal}$ son posibles (Whitcomb et al., 1980). La exactitud $\mathrm{m}_{\mathrm{o}}$ de los gravímetros modernos absolutos se cifra en $\mathrm{m}_{\mathrm{o}}=2 \mu \mathrm{Gal}$ por Niebauer (1995) y Francis (2005). Asumiendo que una tendencia en el valor de la gravedad se puede medir en $\mathrm{N}$ años con la precisión $(12 /(N(N-1)(N+1)))^{1 / 2} \mathrm{~m}_{0}$, es decir, en 10 años con $0,22 \mu \mathrm{Gal} /$ año. Un gravímetro absoluto como el FG5 se considera capacitado para alcanzar incertidumbres por debajo del $\mu \mathrm{Gal}$ en un periodo de 15 años (Van Camp et al., 2005). La gran pregunta a responder cuando se observan cambios de gravedad es qué parte de esos cambios corresponde a movimiento superficial y que parte responde a cambios de masa.

Geológicamente, ninguna parte de la corteza terrestre parece haber estado libre de deformación; en general, la deformación horizontal es del orden de $10 \mathrm{~cm}$ al año y la vertical del orden de algunos $\mathrm{mm}$ al año, proceso que ocurre entre $10^{6}$ y $10^{8}$ años (Lambeck, 1988). Las estimaciones del rebote postglacial en Canadá y Escandinavia muestran variaciones verticales de alrededor de $1 \mathrm{~cm}$ al año, proceso que ha ocurrido en los últimos $10^{4}$ años. Sin embargo, se puede afirmar que existen 
regiones tectónicamente más activas que otras. Por ejemplo, es lógico pensar que si la corteza es más gruesa tenga más estabilidad que en zonas más delgadas (centro de la Península en comparación con el mar de Alborán). En zonas alejadas del límite de las placas tectónicas, zonas intraplaca, la estabilidad tectónica es mayor que en las zonas interplaca, donde se producen la mayoría de los movimientos sísmicos, es decir, las zonas sismotectónicamente activas.

Los movimientos horizontales cosísmicos pueden ser considerables en el caso de megaterremotos y varias veces menores en tierra que en el fondo marino y en el límite de la placa. Para el terremoto de Hosoku-Oki $\left(\mathrm{M}_{\mathrm{w}}\right.$ 9, 2011), mientras que los desplazamientos intersísmicos de la placa pacífica son de $8-9 \mathrm{~cm} /$ año (DeMets et al., 2010), se han medido mediante GPS marino desplazamientos horizontales cosísmicos en el epicentro de entre 5-24 m, y verticales -0,8-3 m (Sato et al. 2011). Los desplazamientos de falla modelados excedieron los $50 \mathrm{~m}$ (Simons et al., 2011). En la costa los desplazamientos horizontales medidos máximos son de 4,4 metros, y la cuantificación vertical en costa casi alcanzó $1 \mathrm{~m}$ de subsidencia (hasta $3 \mathrm{~m}$ en el epicentro (GSI, 2011)), lo cual permitió al agua salvar las defensas anti-tsunami. En la Placa de Nazca los desplazamientos intersísmicos horizontales anuales oscilan entre 12 y 14,5 cm/año (DeMets et al., 2010). Los desplazamientos deducidos de los mecanismos de los terremotos permiten cunatificar desplazamientos horizontales de placa cosísmicos entre 0,6 y 5,5 mm/año entre Europa y África (Buforn et al., 2004).

Los movimientos verticales intersísmicos en la corteza terrestre (levantamiento o subsidencia) son generalmente un orden de magnitud menor que los horizontales. Ocurren en los límites de placas y en el interior de las mismas, siendo las mayores subidas asociadas a tectónica de colisión entre continente-océano o continentecontinente (Lambeck, 1988).

En otros lugares los cambios de gravedad en el muy largo periodo son del orden de $\mathrm{nms}^{-2}$ /año. Goodkind (1986) informa de variaciones pico de $10 \mu \mathrm{Gal}$ en EEUU en varios meses y en Alemania de $2 \mu \mathrm{Gal}$ medidas en unos dos años. Atribuye la diferencia a que en Estados Unidos la actividad sísmica y tectónica es mayor que en el centro del continente europeo.

En la estación de Medicina (Italia) se han obtenido variaciones en gravedad de $+4 \mathrm{nms}^{-2}$ /año (figura 2.6), aunque mediante GPS los valores obtenidos han sido de $-2,57 \pm 0,06 \mathrm{~mm} /$ año (Richter et al., 2004). Existe una diferencia entre ambas técnicas. Las variaciones medidas en Wettzell (Alemania) son de $+4 \mathrm{nms}^{-2} /$ año (figura 7), una vez que han sido eliminadas las señales procedentes del nivel freático y otras de corto periodo. Las variaciones pico a pico que se muestran son en Medicina de $70 \mathrm{nms}^{-2}$ y en Wettzell de casi $200 \mathrm{nms}^{-2}$ están obtenidas mediante un gravímetro absoluto y superconductor. Estas variaciones están probablemente causadas por fenómenos no modelados de período semianual y anual como las variaciones en el nivel freático u otras. 

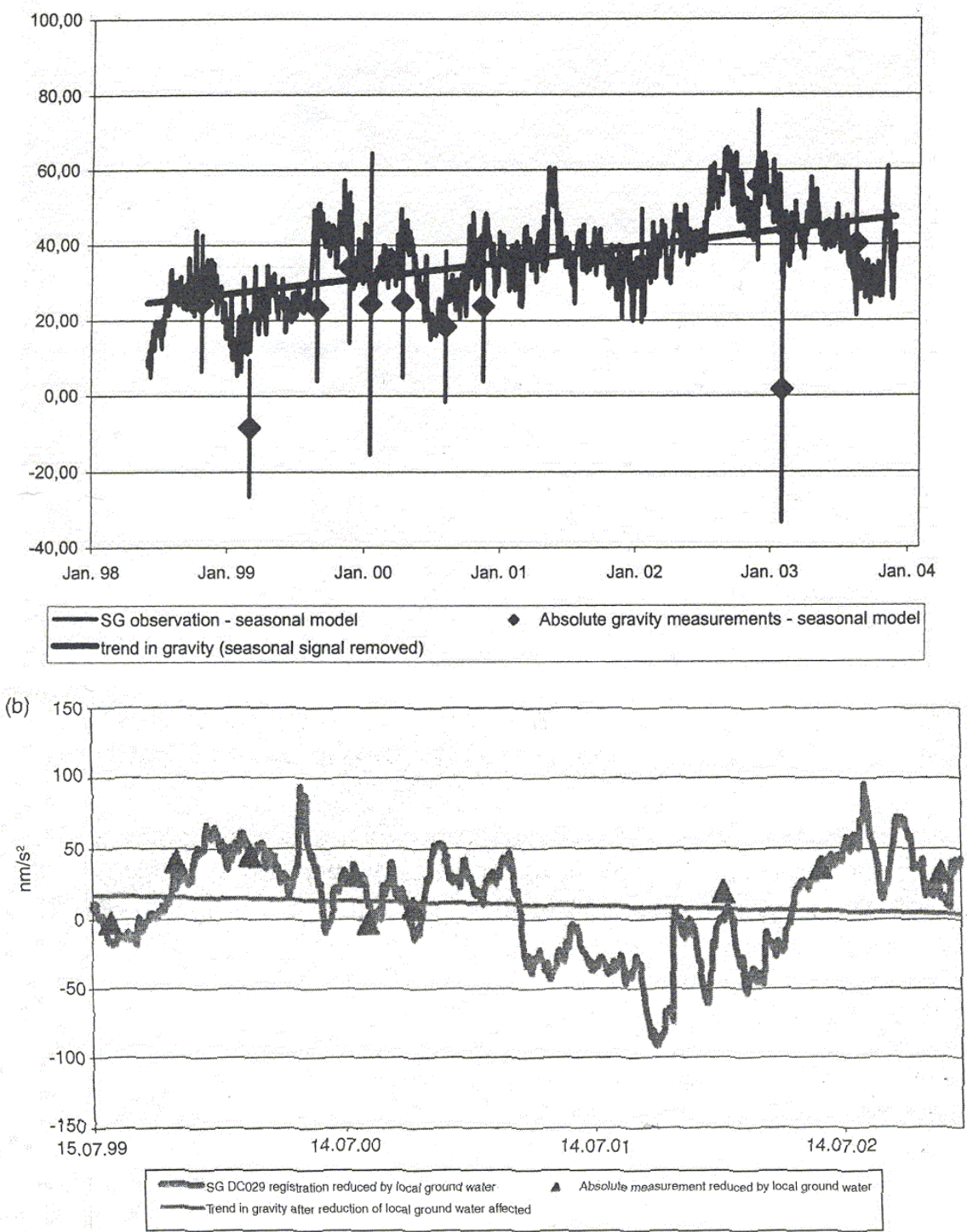

Figura 6. A la izquierda, tendencia lineal de $+4 \mathrm{nms}^{-2}$ en la Medicina (Italia) medida con gravímetros superconductor y absoluto (Richter et al., 2004). A la derecha, medidas en Wettzell (Alemania) con gravímetro superconductor y absoluto una vez eliminado el efecto de variación del nivel freático. Tendencia lineal de $4 \mathrm{nms}^{-2}$ (Richter et al., 2004). 


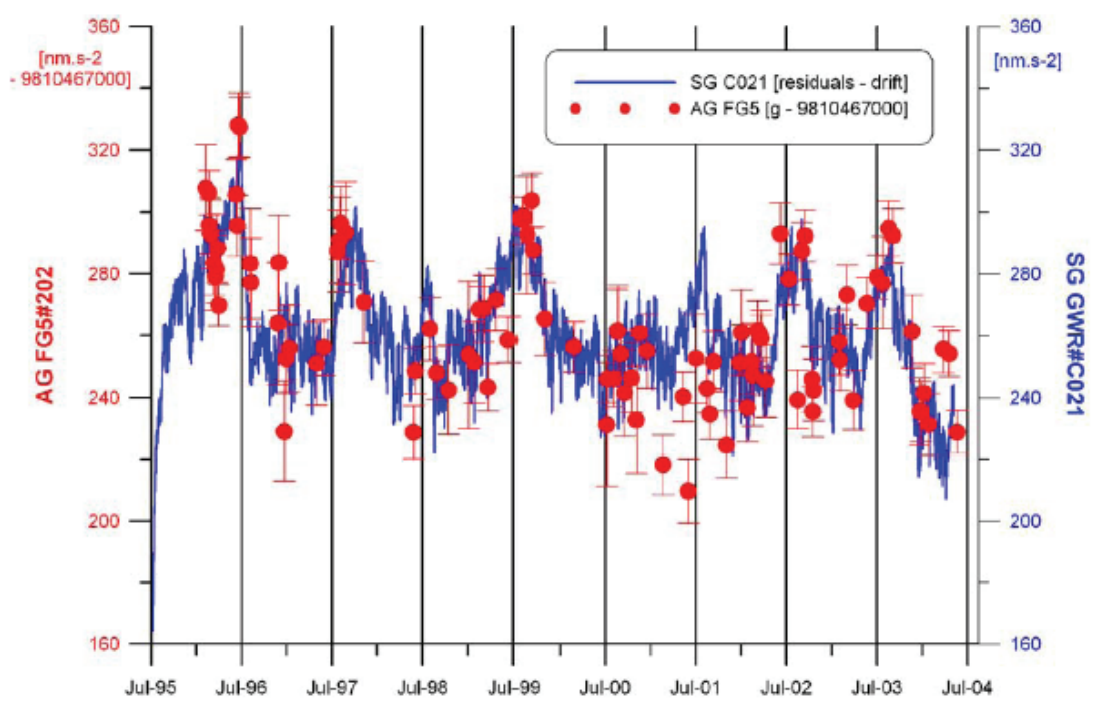

Figura 8. Medidas de ocho años en Membach (Bélgica) con gravímetro superconductor SG C-021 y absoluto FG5-202 una vez eliminados los efectos de mareas terrestres, carga oceánica y atmosférica, movimiento del polo y el efecto de variación del nivel freático. Tendencia lineal de aproximadamente $4.5 \mathrm{nms}^{-2}$ (Van Camp et al., 2004).

En las Árdenas (Bélgica), observaciones con nivelación geométrica han sugerido levantamientos de $1,5 \mathrm{~mm}$ al año o similares, pero con incertidumbres mucho mayores que la propia tasa de deformación. La geología muestra variaciones que difieren en un orden de magnitud, respecto de las medidas de nivelación. El levantamiento observado mediante GPS durante cinco años es $2,7 \pm 0,2 \mathrm{~mm}$ al año en vertical (empleando el software Bernese con procesado en fase de las dos frecuencias GPS disponibles en combinación libre ionosfera, estimando parámetros troposféricos y coordenadas de la estación, en sistema de referencia ITRF97 y estaciones fiduciales cercanas).

La variación de la gravedad medida con gravímetros absolutos da como resultado $-0.43 \pm 0.8 \mu \mathrm{Gal} / \mathrm{año}$, mientras que el superconductor da $-0.6 \pm 0.1$ $\mu \mathrm{Gal} /$ año (Francis et al., 2004; Van Camp et al., 2005). Estas medidas con los gravímetros absolutos y superconductores muestran un levantamiento leve equivalente a unos $3 \pm 0.5 \mathrm{~mm}$ al año. Se compara y se realiza un exhaustivo análisis de los métodos a utilizar cuando las series temporales tienen un número de muestras suficientemente grandes (Van Camp et al., 2005). Las observaciones entre los gravímetros absolutos y superconductores están de acuerdo en el nivel del $\mu \mathrm{Gal}\left(10^{-}\right.$ ${ }^{8} \mathrm{~ms}^{-2}$ ), con variaciones pico a pico de $120 \mathrm{nms}^{-2}$, siendo las discrepancias menores debidas a los efectos geofísicos del entorno no modelados adecuadamente (figura 8). La deriva instrumental del gravímetro superconductor C- 021 de $4.3 \mu \mathrm{Gal}$ al año se evalúa tomando la diferencia entre sus residuos y del gravímetro absoluto FG5202. 
Medidas de gravedad absoluta y GPS sirven para discriminar entre movimientos verticales de la corteza y movimientos de elevación del nivel del mar. En mareógrafos de la región de Gran Bretaña y norte de Francia se ha comparado la gravedad absoluta en un intervalo de una década con el gravímetro FG5-103 con series GPS, sugiriendo una elevación del nivel del mar entre 0,6 y $1,9 \mathrm{~mm} / \mathrm{año}$ (Teferle et al., 2006). En las estaciones de Newlyn y de Lerwick (figura 9) las variaciones de gravedad deducidas han sido de $0,2 \pm 0,2$ y $0,1 \pm 0,2 \mu \mathrm{Gal} / \mathrm{año}$, respectivamente. Las variaciones espaciales en la componente vertical local se obtienen asumiendo un cambio de gravedad de $0,2 \mu \mathrm{Gal}$ por cada mm. Las series muestran variaciones pico a pico de $8 \mu \mathrm{Gal}$ en Lerwick y $6 \mu \mathrm{Gal}$ en Newlyn.

En otro trabajo anterior se obtienen variaciones de gravedad también en Newlyn y Lerwick. Para Aberdeen en cinco años se deduce una tasa de variación de $0,2 \pm 0,6 \mu \mathrm{Gal} /$ año (Williams, Baker y Jeffries, 2001).

Las comparaciones de las tasas obtenidas por serie temporal de gravedad con tasas obtenidas mediante información geológica y las procedentes de modelos de ajuste isostático glacial coinciden en señalar un aumento del nivel del mar de 1,5 $\mathrm{mm} /$ año detectado en estas 7 estaciones de Gran Bretaña y Francia. Sin embargo, a partir de GPS se obtienen distintas estimaciones que oscilan en el rango 0,3-1,7 $\mathrm{mm} / \mathrm{año}$, además de tener una diferencia sistemática con el resto de técnicas comparadas. Diferencias sistemáticas similares han sido descritas por otros autores (Prawirodirdjo y Bock, 2004; Macmillan, 2004). Las discrepancias sistemáticas se asignan a un factor de escala proveniente del centro de masas del sistema de referencia utilizado (Teferle et al., 2006).
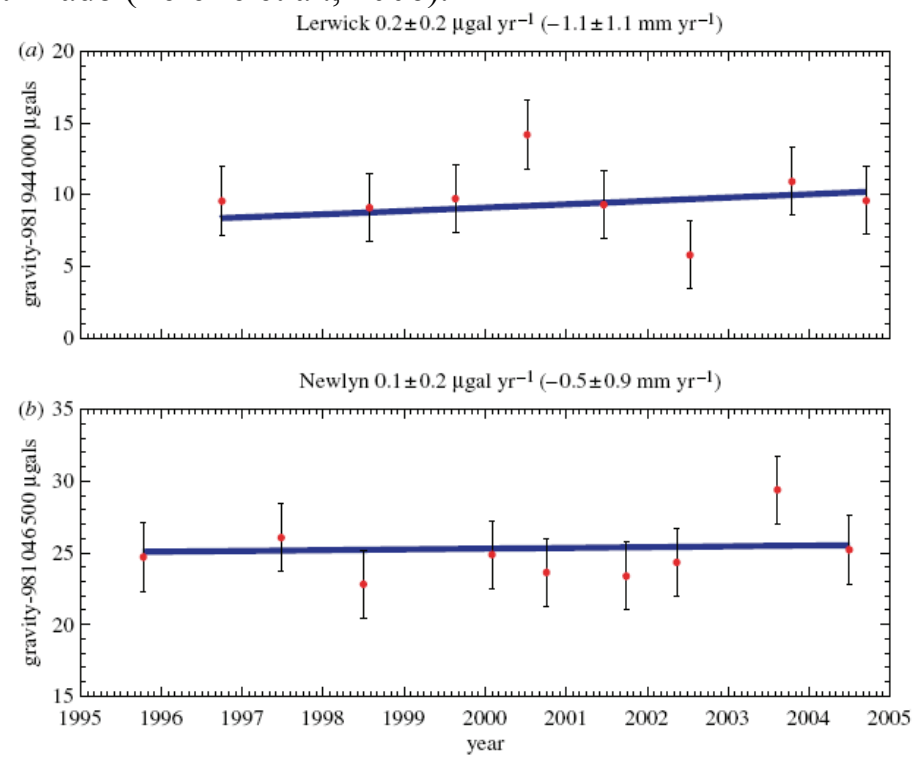

Figura 9. Variaciones temporales de la gravedad en Gran Bretaña a partir de medidas absolutas de la gravedad. La variación en vertical se obtienen asumiendo un cambio de 0,2 $\mu$ Gal por mm (Teferle et al., 2006). 
Las tasas anuales de variación de gravedad inersísmicas son mínimas y menores en comparación a las obtenidas en Europa continental (Wettzell, Medicina, Membach) de las figuras 6,7 y 8 (entre 0,2 y $0,4 \mu \mathrm{Gal} / \mathrm{año}$ ), lo que da cuenta de la gran estabilidad en Europa y de las variaciones esperables en regiones sismotectónicamente estables. En el centro de la Península Ibérica las variaciones son del mismo orden que en Europa Central. La corteza terrestre en las estaciones de gravedad absoluta del Macizo Ibérico (Valle de los Caídos, Madrid, Yebes, Villafranca) muestra una gran estabilidad, con tasa de variación de gravedad del orden entre $+0,2$ y $+0,4 \mu \mathrm{Gal}$ por año (Rodríguez Pujol, 2007), o su equivalente en movimiento vertical entre 0,5 y $1,2 \mathrm{~mm}$ al año, similares a las registradas con series VLBI y GPS. Sin embargo, en el sur de la Península Ibérica a partir de series temporales de gravedad absoluta y relativa, las tasas anuales de variación de gravedad medidas oscilaron entre 0,6 y $3 \mu \mathrm{Gal} / \mathrm{año}$, equivalentes a $2-9 \mathrm{~mm} / \mathrm{año} \mathrm{(Rodríguez} \mathrm{Pujol,}$ 2007).

El posible modelo considerado para los cambios temporales de gravedad de origen geodinámico por movimientos verticales de placas tectónicas es un modelo lineal de la forma:

$$
\Delta g=\Delta g_{m} t+\Delta g_{e}(r, t)+\varepsilon
$$

Donde $\Delta g_{m}$ es la tasa medida, $\Delta g_{e}$ representa las variaciones por nivel freático (periódicas), y $\varepsilon$ otras variaciones de corto y medio período, las dos últimas consideradas como ruido aleatorio en el muy largo plazo.

\section{Cambios locales de gravedad}

Los cambios locales de gravedad pueden estar relacionados con actividad sismotectónica y volcánica en los límites de las placas continentales y también en zonas interiores de la placas. Su extensión se puede cifrar entre 10 y $100 \mathrm{~km}$.

Barnes en 1966 atribuye las variaciones en gravedad observadas antes y después del terremoto de Alaska de 1964 a un incremento neto de la masa total en las regiones epicentrales. Kisslinger (1975) observa levantamientos de $22 \mathrm{~cm}$ entre 1965 y 1966 atribuidos a disminución de densidad en la corteza que relaciona con fenómenos de dilatancia. Efectos de levantamientos de $4 \mathrm{~cm}$ e incrementos de gravedad de $14 \mu \mathrm{Gal}$ entre 1978 y 1981 fueron observados fueron observados por Lambert, Liard y Mainville (1986) en Canadá. Una vez descartados los efectos del rebote postglacial, se atribuyeron a expansión elástica de fracturas los primeros, y a un transporte lateral de agua en el subsuelo el segundo.

Hagiwara (1985) informó de incrementos seculares de $0,02 \mathrm{mGal} /$ año superpuestos con ciclos de tres años con variaciones de 0,04 mGal. También detectó cambios de $-31 \mu \mathrm{Gal}$ en 15 meses, y $15 \mathrm{~cm}$ de movimiento vertical en la Península de Izu (Japón). Cambios de gravedad en diferentes estaciones de California muy diversos se han analizado. Variaciones entre +21 y $-18 \mu \mathrm{Gal}$ se han deducido a partir de varios tipos de instrumentos.

Variaciones no lineales en gravedad pre-, co- y post-sísmicas ( $\mathrm{Li} \mathrm{y} \mathrm{Fu,} \mathrm{1983)}$ aparecen con el terremoto de Tangshan (China) de magnitud 7,8 en agosto de 1976. 
Con variaciones de gravedad alrededor del epicentro y la mayor variación justamente en el epicentro, el campo gravitatorio local se restablece en febrero de 1977, meses después de producirse el evento. Esto induce a no pensar en una intrusión de masa en la corteza, sino en una dependencia de la variación de la gravedad con el estado de esfuerzos en la zona focal. El mecanismo de estas anomalías es explicado por un proceso de dilatación con cuatro fases: fase de compresión (aumento de densidad), dilatación, fase de evento y fase de restauración (figura 10).

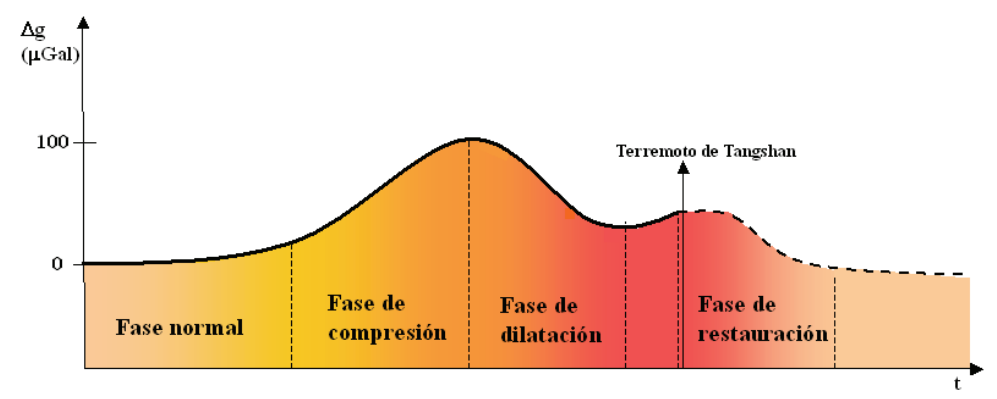

Figura 10. Variaciones teóricas temporales de la gravedad en el modelo de dilatancia (Li y $\mathrm{Fu}, 1983)$.

Son muchos los estudios de variaciones de gravedad relacionados con fenómenos volcánicos. El fenómeno a analizar es altamente dependiente del tipo de volcán y las anomalías de gravedad (junto con otros estudios tomográficos, sísmicos, etc.) pueden ayudar a describir la estructura a la hora de definir un nivel de referencia del volcán a caracterizar. Estructuralmente, anomalías positivas de longitud de onda menores de $20 \mathrm{~km}$ están asociadas, con amplitudes del orden de 30 mGal, a material más máfico y denso que la media del material que lo circunda. Anomalías negativas mayores que $60 \mathrm{mGal}$ se producen en áreas volcánicas más grandes, con materiales piroclásticos altamente silíceos de cenizas y pumitas; también pueden ser debidos a relleno de una caldera no compactada silícea y cuerpos de magma de baja densidad. Cambios pequeños se asocian a respiraderos basálticos de material volátil pobre y a zonas de "rift" extensional, mientras que grandes cambios se refieren a explosivos, estratovolcanes de zonas de subducción y ricos en material volcánico andesítico.

En ciertos casos, en los pasos previos a la inminente erupción volcánica, se producen deformaciones superficiales considerables por la obstrucción de fisuras cercanas a la chimenea volcánica, así como variaciones en el campo gravitatorio próximo al volcán, como ocurrió previamente a la erupción explosiva del volcán del Monte Santa Elena (EEUU) en 1981. Sin embargo, el proceso de alimentación y realimentación con material del manto de la cámara magmática, en los volcanes que la poseen, puede generar variaciones importantes si se realiza la medición en el lugar adecuado. 
En los volcanes activos sin cámara magmática o que se encuentran en zonas de emisión de lava en dorsales oceánicas (por ejemplo Krafla, Islandia) los cambios son grandes y más evidentes. Se distinguen entre variaciones pre-eruptivas y posteruptivas. Para el volcán de Krafla, en las primeras, variaciones de $40 \mu \mathrm{Gal}$ y de 5$10 \mu \mathrm{Gal} / \mathrm{año}$ son factibles. Las segundas detectaron $13 \mu \mathrm{Gal}$ y $-4,5 \mu \mathrm{Gal} / \mathrm{año}$ (Torge, Kanngieser, 1985).Se puede incluso predecir la magnitud de la erupción a partir de los cambios de gravedad precursores. Es de destacar la importancia de las calderas intranquilas y de la medida del gradiente vertical de la gravedad en ellas. Asumiendo el modelo de Mogi, se pueden asociar incrementos de gravedad a lugares lejanos al centro de deformación y también por migración de agua en el sistema hidrotermal del volcán. También se asocian decrementos de gravedad al proceso de drenaje de la cámara de magma poco profunda o a procesos de deflación del volcán (Rymer, 1986).

\section{Conclusión}

Se han mostrado diversos estudios de tasas de variación temporal de la gravedad en los ámbitos global, regional y local. En una zona sismotectónicamente estable las variaciones intersísmicas son menores, mientras que en una zona sismotectónicamente activa pueden ser un orden de magnitud mayor. La gravedad en la superficie terrestre varía de forma temporal secularmente debido a los movimientos verticales de la corteza terrestre, y especialmente, en las regiones que limitan con los bordes de las placas tectónicas. Sin embargo, las tendencias encontradas pueden interpretarse como un movimiento cortical vertical geodinámico, una deformación de la corteza de origen sismotectónico, o un reajuste isostático en la región. Las medidas gravimétricas absolutas, junto con otras técnicas, pueden proporcionar información valiosa sobre los movimientos verticales de la corteza.

\section{Referencias bibliográficas}

BILKER, M., MÄKINEN, J, VIRTANEN, KLOPPING, F., FALK, R., TIMMEN, L., GITLEIN, O. (2004). Time series of gravity in Finland. Gravity, Geoid and Space Missions - GGSM2004, Porto, Portugal.

BJERHAMMAR, A.(1981). The uplift process in Fennoscandia and the corresponding geopotential field from satellites. In Proc. $4^{\text {th }}$ Int. Symp. Geodesy and Physics of the Earth, Karl-Marx-Stadt 1980, Veröff. Zentralinst. F. Physik d. Erde 63, III, 554-581.

BONINI, W.E., LOOMIS, T. P., ROBERTSON, J.D., (1973). Gravity anomalies, ultramafic intrusions, and the tectonics of the region around the Strait of Gibraltar. J. Geophys. Res., $78,8,1372-1382$.

BUFORN, E., BEZZEGHOUD, M., UDÍAS, A., PRO, C. (2004). "Seismic sources on the Iberia-African Plate Boundary and their Tectonic Implications". Pageoph., 161, 623-646. doi:10.1007/s00024-003-2466-1

CHAO, B., GROSS, R. (1987). Changes in the Earth's rotation and low-degree gravitational field induced by earthquakes. Geophys. J. R. Astr. Soc. 91, 569-596.

DE LINAGE, C., ET AL. (2009). Separation of coseismic and postseismic gravity changes for the 2004 Sumatra-Andaman earthquake from $4.6 \mathrm{yr}$ of GRACE observations and 
modeling of the coseismic change by normal-modes summation. Geophys. J. Int. 176, 695-714.

doi: 10.1111/j.1365-246X.2008.04025.x

DEMETS, C., GORDON, R.G., ARGUS, D. (2010). Geologycally current plate motions. Geophys. J.Int.181, 1-80.

doi:10.1111/j.1365-246X.2010.04562.x

FRANCIS, O. (2005). Results of the International Comparison of Absolute Gravimeters in Walferdange (Luxembourg). Gravity, Geoid and Space Missions GGSM2004. Int. Ass. Geodesy. Porto.

GEOSPATIAL INFORMATION AUTHORITY OF JAPAN. http://www.gsi.go.jp/cais/topic110421-index-e.html (2011).

GIMÉNEZ, J., SURIÑACH, E., GOULA, X., CATURLA, J.L. (1993). Análisis de datos de Nivelación de Alta precisión. Valoración de su posible aplicación al estudio de los movimientos vericales en el noroeste de la Península Ibérica. Boletín de Información Servicio Geográfico del Ejército (SGE), 75.

GIMÉNEZ, J., SURIÑACH, E., GOULA, X. (2000). Quantification of vertical movements in the eastern Betics (Spain) by comparing levelling data. Tectonophysics, 317, 237-258. doi:10.1016/S0040-1951(99)00318-2

GIMÉNEZ, J., SURIÑACH, E., FLETA, J., GOULA, X. (1996). Recent vertical movements from high precision levelling data in northeast Spain. Tectonophysics, 263, 149-161.

doi:10.1016/S0040-1951(96)00037-6

GOODKIND, J.M. (1986). Continous measurements of non tidal gravity variations. $J$. Geophys. Res., 91, 9125-9134. doi:10.1029/JB091iB09p09125

HAGIWARA, Y. (1985). Gravity changes in the Izu peninsula. In Earthquake Prediction Research vol. 3, 3-4.

HEKI,K., MATSUO, K. (2010). Coseismic gravity changes of the 2010 earthquake in central Chile from satellite gravimetry. Geophys. Res. Lett., 37, L24306. doi:10.1029/2010GL045335

JACOBY, W. L., SEIDLER, E. (1981). Plate kynematics and the gravity field. Tectonophysics, 74, 155-167.KISSLINGER Y T. RIKITAKE EDS. AMSTERDAM.

LAMBERT, A. ET AL. (2001). New constraints on Laurentide postglacial Rebound from Absolute Gravity measurements. Geophys. Res. Lett., 28, 10, 2109-2112. doi:10.1029/2000GL012611

LI, R., FU, Z. (1983). Local gravity variations before and after the Tangshan Earthquake $(\mathrm{M}=7.8)$ and the dilatation process. Tectonophysics, 97, 159-169. doi:10.1016/0040-1951(83)90143-9

LIU, X., (1993). Krustenaufbau und Tektonik des westlichen Mittelmeeraumes abgeleitet aus seismischen und gravimetischen Daten. Universität Hamburg.

KISSLINGER, C. (1975). Processes during the Matsushiro, Japan earthquake swarm as revealed by levelling, gravity, and spring-floor observations, Geology, 3, 57-62.

KUO, J. T., SUN, Y. (1993). Modeling gravity variations caused by dilatancies. Tectonophysics, 227, 127-143.

doi:10.1016/0040-1951(93)90091-W 
LAMBECK. K. (1988). Geophysical Geodesy. The slow deformation of the earth. Oxford University Press.

LAMBECK, K., JOHNSTON, P., NAKADA, M. (1990). Holocene glacial rebound and sea level change in NW Europe. Geophys. J. Int., 103, 451-468.

LAMBERT, A., LIARD, J. O., MAINVILLE, A. (1986). Vertical movement and gravity change near the La Grande-2 reservoir, Quebec. J. Geophys. Res., 91, B9, 9150-9160. doi:10.1029/JB091iB09p09150

JACHENS, R. C., ROBERTS, C. W. (1985). Temporal and areal gravity investigations at long Valley Caldera, California. J. Geophys. Res., 90, 11210-11218. doi:10.1029/JB090iB13p11210

MÄKINEN, J., A. ENGFELDT, B. G. HARSSON, H. RUOTSALAINEN, G. OJA, T. WOLF, D. (2005).The Fennoscandian land uplift gravity lines 1966-2003. Gravity, Geoid and Space Missions GGSM2004. Int. Ass. Geodesy. Porto.

MÄKINEN, J., ALMAVICT, M., K. SHIBUYA, Y. FUKUDA (2007). Absolute gravimetry in Antarctica: Status and prospects. J. Geodynamics, 43, 339-357.

doi:10.1016/j.jog.2006.08.002

MILNE, G.M., DAVIS, J. L., MITROVICA, J. X., SCHERNECK, H.-G., JOHANSSON, J. M., VERMEER, M., KOIVULA, M. H. (2001). Space-Geodetic Constraints on Glacial Isostatic Adjustment in Fennoscandia, Science, 291, 2381. doi: 10.1126/science. 1057022

MORELLI, C., PISANI, M., GANTAR, C. (1975). Geophys. anomalies and Tectonics in the Western Mediterranean. Boll. Geof. Teor. ed appl., XVII, 67, 211-249.

MORELLI, C., NICOLICH, R. (1980). The structure of the western Mediterranean basins from marine geophysical data. In Evolution and tectonics of the western Mediterranean and surrounding areas. Special publication no.201. European Geophys. Society, Viena 12-14 de septiembre de 1979. Instituto Geográfico Nacional. Madrid.

NIEBAUER, T.M., SASAGAWA, G. S., FALlER, J. E., KLOPPING, F. (1995). A new generation of absolute gravimeters. Metrologia, 1995, 32, 159-180. doi:10.1088/0026-1394/32/3/004

NIKOLAIDIS, R. (2002). Observation of geodetic and seismic deformation with the Global Positioning system. PhD Thesis. University of California. San Diego.

PRAWIRODIJO, L., BOCK, Y. (2004). Instantaneous global plate motion model from 12 years of continuous GPS observations. J. Geophys. Res. 109, B08405. doi:10.1029/2003JB002944

RICHTER, B., ZERBINI, S., MATONTI, F., SIMON, D. (2004). "Long term crustal deformation monitored by gravity and space techniques at Medicina, Italy, and Wetzell, Germany". J. Geodyn., 38, pp. 281-290.

doi:10.1016/j.jog.2004.07.013

RODRÍGUEZ PUJOL, E. (2005). Absolute Gravity network in Spain. Física de la Tierra Vol. 17, 2005, 147-163.

RODRÍGUEZ PUJOL, E. (2007). Estudio de movimientos verticales de la corteza terrestre en el sur de la Península Ibérica a partir de medidas absolutas y relativas de la gravedad. Tesis Doctoral. Universidad Complutense.

RUNDLE, J. B., WHITCOMB (1986). Modeling gravity and trilateration data in Long Valley, California, 1983-1984. J. Geophys. Res., 91, 12675-12682.

doi:10.1029/JB091iB12p12675 
RYMER, H., BROWN, G. C. (1986). Gravity fields and the interpretation of volcanic structures: geological discrimination and temporal evolution. J. Volcanol. Geotherm. Res., 27, 229-254. doi:10.1016/0377-0273(86)90015-6

SASAGAWA, G., ZUMBERGE M. (1991). Absolute Gravity Measurements in California, 1984-1989. J. Geophys. Res., 96, B2, 2501-2513. doi:10.1029/90JB02283

SATO, T., OKUNO, J., HINDERER, J., MACMILlAN, D, PLAG, H., FRANCIS, O., FALK, R., FUKUDA, Y. (2006). Secular displacement and gravity rates in Ny-alesund, Svalbard in the Arctic-effects of post-glacial rebound and present-day ice melting. Geophys. J. Int., 165, 729-743. doi: $10.1111 /$ j.1365-246X.2006.02992.x

SATO, M. ET AL. (2011). Displacement above the Hypocenter of the 2011 Tohoku-Oki. Science 332 (6036), 1395. doi:10.1126/science.1207401.

SEEBER, G. (1993). Satellite Geodesy. De Gruyter.

SURIÑACH, E., UDÍAS, A. (1978). Determinación del espesor de la Corteza en las Béticas. Tesis doctoral. Universidad Complutense de Madrid.

SIMONS, M. ET AL. (2011).The 2011 M 9.0 Tohoku-Oki Earthquake: Mosaicking the Megathrust from seconds to centuries. Science 332, 1421-1425. doi:10.1126/science.1206731.

SCHEIDEGGER, A.E. Principles of Geodynamics.Springer-Verlag. New York. 1982.

SCHOLZ, C. H. (1972). Crustal movements in tectonics areas. Tectonophysics, 14,201-217.

TEFERLE, F. N., BINGLEY, R. M., WILLIAMS, S.D.P., BAKER, T. F., DODSON, A. H. (2006). Using continuous GPS and absolute gravity to separate vertical land movements and changes in sea-level at tide-gauges in the UK. Phil. Trans., Roy. Soc. London, A, 364, 917-930.

doi:10.1098/rsta.2006.1746

TORGE, W., KANNGIESER. (1985). Journal of Geophysical Research, 90, 10173-10177.

TORGE, W. (1989). Gravimetry. Springer. Berlin.

TORGE, W. (2001). Geodesy. Springer. Berlin.

TORNÉ, M, FERNÁNDEZ, M., COMAS, M.C., SOTO, J. I. (2000). Lithospheric structure beneath the Alboran Basin: Results from 3D Gravity Modeling and tectonic relevance. $J$. Geophys. Res., 105, B2, 3209-3228.

UDÍAS, A. (1999). Principles of Seismology. Cambridge University Press.

VAŃIČEK, P., KRAKIWSKY, E. (1986). Geodesy: The concepts. North Holland.Amsterdam.

VAN CAMP, M., WILLIAMS, S., FRANCIS, O. (2005). Uncertainty of absolute gravity measurements. J. Geophys. Res., 110, B05406.

doi:10.1029/2004JB003497

WHITCOMB, J. H., FRANZEN, O. F., GIVEN, J. W., PECHMANN, J. C., RUFF, L. J. (1980). Time-Dependent Gravity in southern California, May 1974 to April 1979. J. Geophys. Res., 85, 4363.

WILLIAMS, S. D. P., BAKER T. F., JEFFRIES G. (2001). Absolute gravity measurements at UK tide gauges. Geophys. Res. Lett., 28,12, 1109-1112.

doi:10.1029/2000GL012438 